3

4

5

6

7

9

10

11

\title{
Aging Mechanism of SBS Modified Asphalt Based on Chemical
}

Reaction Kinetics

$$
\text { Yuanyuan Wang }{ }^{1} \quad \text { Lu Sun }{ }^{2,3} \quad \text { Yuxiu Qin }^{4}
$$

${ }^{1}$ School of Transportation, Southeast University

No.2 Sipailou, Nanjing, 210096, China

Office: (025)8379-2619, Email: wangguangyuan-1005@163.com

${ }^{2}$ School of Transportation, Southeast University

No.2 Sipailou, Nanjing, 210096, China

Office: (202)319-6671; Email: $\underline{\text { sunlu@ @ua.edu }}$

(1)

(1)

${ }^{3}$ Department of Civil Engineering

The Catholic University of America, Washington, DC 20064, USA

Email: $\underline{\text { sunlu@cua.edu }}$

${ }^{4}$ School of Transportation, Southeast University

No.2 Sipailou, Nanjing, 210096, China

Office: (025)8379-2619, Email: 976032157@qq.com

1

C 2015. This manuscript version is made available under the Elsevier user license http://www.elsevier.com/open-access/userlicense/1.0/ 
1 Abstract: Research on aging mechanism of SBS modified asphalt can provide

2 theoretical and technical support not only for the choice of asphalt pavement materials

3 but also for recycling of SBS modified asphalt pavement. Dynamic equations of

4 asphalt under different aging conditions were respectively established according to

5 chemical reaction kinetics. And two kinds of micro analysis methods, component

6 analysis and GPC (gel permeation chromatography), were used to analyze the change

7 of asphalt composition and asphalt molecular structure during the aging process. In

8 addition, the correlation between activation energy and asphalt microscopic

9 components, asphalt molecular structure was analyzed through grey entropy theory

10 analysis method. Different from previous research, the results demonstrate that the

11 activation energy of asphalt is not a constant index. SBS network structure loses

12 gradually due to the degradation of SBS modifier during the aging process. SBS

13 modified asphalt after short-term aging degrades to more medium molecular size

14 structure, which makes the activation energy become lower and improves asphalt

15 temperature sensitivity. However, the SBS modifier after long-term aging has almost

16 degraded completely, which increases the activation energy and makes asphalt

17 temperature sensitivity approximate to base asphalt. The results of grey entropy

18 theory analysis show that the activation energy will be reduced with the decrease of

19 saturate content, the increase of medium molecular size structure content and resins

20 content.

21 Keywords: SBS modified asphalt, chemical reaction kinetics, aging mechanism, component analysis, Gel Permeation Chromatography 


\section{Introduction}

Asphalt aging has been recognized as an important aspect in asphalt research. Asphalt binder aging occurs during the mixing and construction process as well as during service process in the road. Due to the oxidation, the aging process will result in the loss of volatile components and reorganization of asphalt binder molecules. The aging of asphalt binder is an oxidative chemical process that will lead to the formation of carbonyl compounds and sulfoxides. Then, the stiffness, adhesion and flexibility properties of binder will be affected [1]. Consequently, aging of asphalt binder has a direct impact on road performance. During the service process of asphalt pavement, the asphalt will become stiffer and more brittle due to oxidation, sunlight and ultraviolet radiation. However, the oxidation is the main factor. More importantly, asphalt peeling off from aggregate surface, pumping slurry, loose, threshing forming pit slot and water seeping into middle or lower layer can also cause water damage. All these will influence the performance and service function of asphalt pavement significantly [2-4]. Therefore, understanding the aging mechanism of asphalt can have a significant role in pavement maintenance, choosing the asphalt materials, strengthening durability of the pavement, and recycling SBS modified asphalt pavement that will save resources and reduce the pressure of environment.

Owing to the increasing traffic, ordinary asphalt pavement has been gradually unable to meet the needs of transportation and environment. Recently, researches and engineering practices have shown that SBS ( styrene-butadiene-styrene block copolymers ) modifier can comprehensively improve pavement performance[5]. 
1 Nowadays, a number of freeways have required rehabilitation or reconstruction in

2 China, which produces a lot of aged SBS modified asphalt mixture. The abandonment

3 of these materials not only wastes resources, but also causes environmental pollution

4 and excessive exploitation of new natural resources [6]. Therefore, recycling of the

5 aged SBS modified asphalt mixture has became increasingly priority in the highway

6 industry.

7 Though researchers have paid more attention to the aging mechanism of SBS

8 modified asphalt. However, studies mainly focused on evaluating and analyzing the

9 change of performance indicators before and after aging from the superficial

10 phenomenon. For instance, Airey et al. carried out a large amount of experimental

11 research for SBS modified asphalt after RTFOT and PAV aging and discussed the

12 differences of aging performance between SBS modified asphalt and base asphalt [7].

13 The results indicated that the aged asphalt containing more modifiers showed more

14 viscous characteristics rather than usually imagined elastic characteristics. And the

15 transformation to viscous flow state for SBS modified asphalt was mainly due to the 16 degradation of SBS modifiers.

17 Paling et al. evaluated the influence of different aging states on asphalt high 18 temperature performance, low temperature crack resistance and temperature 19 sensitivity through penetration, penetration index, ductility, softening point, etc. test 20 indexes. The results showed that the addition of SBS modifiers significantly improved 21 the anti-aging property of asphalt [8].

22 Siddiqui et al. studied the performance changes of aged SBS asphalt using 
1 infrared spectra and thermal analysis, which showed the addition of SBS modifiers

2 into asphalt led to better anti-aging performance. Meanwhile, they tried to recycle

3 modified asphalt by adding new asphalt. Test results indicated that the recycling

4 technology of modified asphalt was feasible [9].

5 In order to evaluate the rheological properties and the viscosity-temperature 6 characteristics of asphalt/polyethylene blends, Vargas et al. obtained the activation 7 energy by solving the chemical reaction kinetics equation. And they acknowledged 8 that lower activation energies are preferred because viscosity changes slightly with 9 changing the same temperature amplitude [10]. Mturi et al. compared the differences of rheological properties between aged base asphalt binder and aged modified asphalt binder through rotational and oscillatory-derived viscosities test. The changes of aging 12 rate were also analyzed by Arrhenius relation based on chemical reaction kinetics equation. They found that the Arrhenius relation can well describe the changes of 14 aging rate for the accelerated thermal ageing methods [11]. Vargas and Mturi both treated the activation energy as a constant. However, aging influenced asphalt binder chemistry and rheology significantly. These changes contain increases in amount of large molecules, transformation of generic fractions and the formation of carbonyl compounds and sulfoxides [12]. All these changes will lead to the change of asphalt binder microscopic components and molecular structures, which will cause the changes of activation energy and make the activation energy is no longer a constant 21 during the aging process.

22 Herrington et al. tested the changes in carbonyl area and verified the aging rate 
1 meets the assumption of a first order dependence on oxygen concentration for rolling

2 thin film oven test and pressure aging vessel. The establishment of this assumption

3 proved the correctness of Arrhenius relation used to analyze aging rate during the

4 process of rolling thin film oven and pressure aging vessel [13]. Xin, et al. analyzed

5 the relationship between viscosity and carbonyl area tested by Fourier transform

6 infrared (FTIR) spectroscopy during oxidation aging process. A strong correlation is

7 observed between the rheological measurements and chemical analyses [14]. This

8 strong correlation demonstrated that the chemical composition and the rheological

9 properties of asphalt binder are complementary to each other. The flow state of

10 asphalt binder will be influenced by the variation of chemical composition during

11 oxidation aging process. It provides a strong argument for the Arrhenius relation

12 based on rheological properties. Therefore, we can use the flow state to characterize

13 the aging conditions of asphalt binder. The Arrhenius relation combined chemical

14 reaction kinetics with the principle of time temperature conversion, which was used to

15 analyze the aging mechanism in this study.

16 Research on aging mechanism of SBS modified asphalt can give a direction for

17 recycling of SBS modified asphalt pavement, so as to reduce the discard of waste SBS

18 modified asphalt mixture, save resources and protect the environment. In this study,

19 asphalt aging kinetics model was presented to further reveal the aging mechanism of

20 SBS modified asphalt and understand aging process from the perspective of chemical

21 reaction kinetics theory. The differences of aging mechanism between base asphalt

22 and SBS modified asphalt were also analyzed through micro components analysis and 
1 gel permeation chromatography analysis.

2

\section{2. Chemical reaction kinetics}

4 Chemical reaction kinetics is a branch of physical chemistry discipline and its 5 research object is a dynamic system. Reaction rate as an important indicator in

6 chemical reaction kinetics was used to measure the speed level of chemical reaction.

7 The relationship between chemical reaction rate and concentration of components is

8 calculated by Eq.(1) [15].

9

10

$$
r=f\left(C_{A}, C_{B} \cdots C_{I} \cdots\right)
$$

Where $\mathrm{r}$ is reaction rate, mol.(L.h $)^{-1} ; \mathrm{C}_{\mathrm{I}}$ is the concentration of I component, mol/L. For most simple reactions and some compound reactions, the reaction rate equation is expressed as follows:

$$
r=k C_{A}{ }^{p} C_{B}^{q} \cdots
$$

Where $\mathrm{k}$ is chemical reaction rate constant, $\mathrm{h}-1 ; \mathrm{p} 、 \mathrm{q} . .$. is respectively the reaction orders of corresponding components A、B... The sum of each component reaction orders, $\mathrm{n}$, is called total orders of reactions. And the reaction whose total orders is simple positive integers and zero is called as simple orders reaction. Usually asphalt aging can be thought as one orders reaction, which means asphalt aging rate is in direct proportion to the first power of reactants concentration, Eq. (3) $[13,16]$ :

$$
-\frac{d c}{d t}=k c
$$

Where $\mathrm{c}$ is component concentration, mol/L; t is reaction time, h. And Eq. (4) can be obtained through solving Eq. (3). 


$$
\ln c=B-k t
$$

2 Where $\mathrm{B}$ is the integral constant. For the simple series reaction, its reaction rate

3 equation can be easily established when the reaction series $\mathrm{n}$ and the reaction rate

4 constant $\mathrm{k}$ are known. But the reaction rate constant $\mathrm{k}$ for one series reaction can be

5 obtained from drawing lnc to $\mathrm{t}$, and then the straight slope is equal to $-\mathrm{k}$.

6 In order to describe the relationship between reaction temperature $\mathrm{T}$ and reaction

7 rate constant $\mathrm{k}$, the concept of activation energy is put forward and chemical reaction

8 kinetics empirical formula is established by Arrhenius, Eq. (5).

9

$$
\ln k=\ln A-\frac{E_{a}}{R T}
$$

Where $\mathrm{A}$ is pre-exponential factor or frequency factor constant; $\mathrm{R}$ is molar gas constant, about $8.314 \mathrm{~J} /(\mathrm{mol} . \mathrm{K})$; $\mathrm{T}$ is absolute temperature, $\mathrm{K}$; Ea is activation energy, $\mathrm{J} / \mathrm{mol}$.

According to the principle of chemical reaction kinetics, the activation energy refers to the energy barrier about activation of molecular. In Arrhenius's point of view, activation energy is the energy needed to transform the reactant molecules into activated molecules [17]. To some extent, the activation energy reflects the molecular composition and structure of reactants, which decides the chemical reaction rate. Eq. (5) also indicates that there are some connections between activation energy and temperature sensitivity. It reflects how much the chemical reaction rate changes when the test temperature increases or reduces to a certain degree. Generally, greater activation energy corresponds to a smaller chemical reaction rate at a given temperature. However, for the temperature sensitivity, the greater the activation 
1 energy is, the worse the temperature sensitivity in a certain temperature interval is.

2 Better temperature sensitivity means that the viscosity slightly with variation of the

3 same temperature amplitude. Lower activation energies are preferred because the

4 change of viscosity is smaller with changing the same temperature amplitude

5 according to Equation (6).

6 The temperature dependence of the asphalt viscoelastic behavior, as indicated by

7 the shift factors determined from the construction of master curves, may fit either the

8 Arrhenius or the Williams-Landel-Ferry (WLF) equation. Within the temperature

9 range studied in this work, the temperature dependence of the shift factor for all the

10 systems studied is described by an Arrhenius-type equation fairly well, Eq.

11 (6).According to the principle of time temperature conversion, the shift factor $\alpha(\mathrm{T})$ is

12 defined as Eq. (7) [18].

13

14

$$
\begin{gathered}
\alpha(T)=\exp \left[\frac{E_{a}}{R}\left(\frac{1}{T}-\frac{1}{T_{0}}\right)\right] \\
\lg \alpha(T)=\lg \mathrm{f}_{\mathrm{r}}-\lg f_{0} \quad \alpha(T)=\frac{f_{r}}{f_{0}}
\end{gathered}
$$

where $\alpha(T)$ is the shift factor at a temperature T relative to the reference temperature $T_{0} ; f_{r}$ is the shift frequency; $f_{0}$ is the frequency reference.

The complex modulus of asphalt under different temperature and frequency were obtained through frequency domain analysis. The principal curve of asphalt complex modulus was drawn based on the principle of time temperature conversion. The shift factor $\alpha(T)$ could be obtained by the principal curve according to Eq.(7). Then, a straight line could also be obtained by drawing a figure $\ln \alpha(\mathrm{T})$ to 1/T. Comparing Eq. 
1 (5) with Eq. (6), it can be found that the straight slope is equal to $\mathrm{Ea} / \mathrm{R}$ and the

2 intercept equals to $-\mathrm{Ea} /\left(\mathrm{RT}_{0}\right)$. So chemical reaction kinetics parameters based on the

3 complex modulus of asphalt can be obtained. Then the dynamic characteristics of

4 asphalt were analyzed during aging process according to chemical reaction kinetics.

5

6

7

\section{Material preparation}

Liaohe AH90 oil bitumen (an 80/100 pen grade bitumen, PG 64-22) and 791-SBS modifiers (one kind of SBS block copolymer modifiers and belongs to the linear structure with the ratio of styrene and butadiene being 30:70.) were selected as raw materials. The ductility at $5^{\circ} \mathrm{C}$ of asphalt binder after long-term aging process as the judge index was used to assess the SBS modifier decomposition conditions. If the ductility test at $5^{\circ} \mathrm{C}$ is hardly to proceed and the test specimens fracture directly, we think the SBS modifier degrade completely. In order to guarantee the SBS modifier to degrade completely during aging test, the SBS modifier content of modified asphalt was identified as $3 \%$ through indoor ductility test verification. The preparation steps are as follows. Firstly, add 3\% (mass fraction) SBS modifier and a small amount of stabilizer into the melting base asphalt AH90. Then, at temperature of $170^{\circ} \mathrm{C}$, the modified asphalt is subject to high speed shearing for $30 \mathrm{~min}$ and left to develop for 1 h. The basic performance indicators of AH90 oil bitumen and SBS modified asphalt (PG 70-22) are shown in Table 1.In order to compare similarities and differences between base asphalt and SBS modified asphalt, aged AH90 asphalt and aged SBS modified asphalt were respectively prepared by carrying out laboratory short-term and 
1 long-term aging process: rolling thin film oven test (RTFOT, standard ASTM D2872)

2 and pressure aging vessel (PAV, standard ASTM D6521).

3

Table1. Test results of base asphalt and SBS modified asphalt

\begin{tabular}{|c|c|c|c|c|c|c|c|c|}
\hline Tests & $\begin{array}{l}\text { Penetration } \\
\left(25^{\circ} \mathrm{C}\right) \\
(0.1 \mathrm{~mm})\end{array}$ & $\begin{array}{l}\text { Softening } \\
\text { Point } \\
\left({ }^{\circ} \mathrm{C}\right)\end{array}$ & $\begin{array}{l}\text { Softening } \\
\text { point } \\
\text { difference } \\
\left({ }^{\circ} \mathrm{C}\right)\end{array}$ & $\begin{array}{l}\text { Ductilit } \\
\mathrm{y} \\
(\mathrm{cm})\end{array}$ & $\begin{array}{c}\text { Viscosity } \\
\left(135^{\circ} \mathrm{C}\right) \\
(\mathrm{mPa} . \mathrm{s})\end{array}$ & $\begin{array}{l}\mathrm{G}^{*} / \sin \delta \\
\left(64^{\circ} \mathrm{C}\right) \\
(\mathrm{KPa})\end{array}$ & $\begin{array}{l}\text { Creep } \\
\text { stiffness } \\
\mathrm{S}\left(-18{ }^{\circ} \mathrm{C}\right. \\
)(\mathrm{MPa})\end{array}$ & $\begin{array}{l}\text { Creep } \\
\text { rate } \\
\mathrm{m} \\
\left(-18^{\circ} \mathrm{C}\right)\end{array}$ \\
\hline Test methods & ASTM D 5 & $\begin{array}{l}\text { ASTM } \\
\text { D } 36\end{array}$ & $\begin{array}{l}\text { ASTM } \\
\text { D5976 }\end{array}$ & $\begin{array}{l}\text { ASTM } \\
\text { D113 }\end{array}$ & $\begin{array}{c}\text { ASTM D } \\
4402\end{array}$ & $\begin{array}{c}\text { AASHT } \\
\text { O T } \\
315 \\
\end{array}$ & ASTM D & 5648 \\
\hline AH90 & 84 & 42.5 & - & $\begin{array}{l}>100 \\
\left(15^{\circ} \mathrm{C}\right)\end{array}$ & 410 & 1.1 & 294 & 0.34 \\
\hline $\begin{array}{l}\text { SBS } \\
\text { modified } \\
\text { asphalt }\end{array}$ & 74 & 51.5 & 1 & $41\left(5^{\circ} \mathrm{C}\right)$ & $\begin{array}{l}1435 \text { (shea } \\
\mathrm{r} \text { thinning) }\end{array}$ & 2.1 & 267 & 0.35 \\
\hline
\end{tabular}

4

5 4. Testing methods

6

\subsection{Dynamic shear rheology test (AASHTO T 315)}

7 respectively. According to AASHTO MP1 test method, $1 \mathrm{~mm}$ thickness asphalt sample was placed in the middle of two parallel disc with the dimension of $25 \mathrm{~mm}$. Complex modulus $\left(\mathrm{G}^{*}\right)$ and phase angle $(\delta)$ were calculated through measuring the applying torque with the bottom plate fixed and the upper plate moving at different shear 
1 frequencies[19]. The strain control mode was used in this test.

2

\section{$3 \quad 4.2$ Component analysis}

4 colloid structure [20]. asphalt.

The appropriate component contents are the basic guarantee of asphalt performance. To explain the results of chemical reaction kinetics, component analysis method was used to study the change of each component of asphalt under different aging conditions. The components of AH90 asphalt and SBS modified asphalt were analyzed by means of four component analysis based on technical specification (standard ASTM D4124). Asphalt colloidal unstable index, $\mathrm{I}_{\mathrm{c}}$, is calculated according to Eq. (8). Colloidal unstable index reflects the stability of structural system of asphalt performance. A greater colloidal unstable index corresponds to a more unstable

$$
I_{c}=\left(S+A_{s}\right) /\left(R+A_{r}\right)
$$

where $\mathrm{S}(\%)$ is the saturates content of asphalt, $\mathrm{A}_{\mathrm{s}}(\%)$ is the asphaltene content of asphalt, $\mathrm{R}(\%)$ is the resins content of asphalt, and $\mathrm{A}_{\mathrm{r}}(\%)$ is the aromatics content of

\subsection{Gel permeation chromatography (GPC)}

GPC is a method of separation according to the molecular size when polymer solution goes through a column composed of special porous fillers. GPC analysis is commonly used to determine the molecular weight and molecular weight distribution.

Gel permeation chromatography analyzer is mainly composed of solvent, solvent pump storage, solution injection control valve, GPC column, detector, waste solution 
1 and solvent storage pool, etc $[21,22]$. In order to better explain the analysis result of

2 chemical reaction kinetics, Malvern ViscotekHT-GPC type gel chromatography

3 permeameter is adopted to analyze the distribution of micro molecular of asphalt

4 under different aging conditions.

5 Most polymers are composed of different size molecules with the same link 6 structure, but with different molecular chain length. The molecular weight of polymer

7 is actually a statistical average of molecular weight of a variety of different sizes

8 polymer. Each polymer has its own molecular weight distribution due to the poly

9 dispersion of molecular weight. Common average molecular weight contains

10 number-average molecular weight, $M_{n}$, and weight-average molecular weight, $M_{w}$,

11 Eqs. (9 and10). Distribution width of polymer molecular weight is a quantitative

12 description of polymer dispersing degree, which is described using dispersion $M_{w} / M_{n}$

13 [23]. From the results of GPC, we can obtain the distribution of polymers with

14 different molecular weight according to leaching volume. Because the mole fraction is

15 proportional to the leaching volume for the polymer solution, $M_{n}$ and $M_{w}$ can be

16 automatically calculated by the computer based on the leaching volume and the

17 leaching net mass at different time [24].

$$
\begin{gathered}
M_{n}=\frac{\sum n_{i} M_{i}}{\sum n_{i}}=\sum N_{i} M_{i} \\
M_{w}=\frac{\sum w_{i} M_{i}}{\sum w_{i}}=\sum W_{i} M_{i}
\end{gathered}
$$

where $\mathrm{n}$ is mole number of molecular in mol, w is the weight of molecular in $\mathrm{g}, \mathrm{M}$ is

21 molecular weight in $\mathrm{kD}, \mathrm{N}$ is the mole fraction and $\mathrm{W}$ is the weight fraction. 


\section{$2 \quad 4.4$ Grey entropy theory analysis}

3 Grey entropy correlation analysis, as an important part of grey theory system, is an

4 effective method for information system analysis. It is also the cornerstone of the grey

5 system analysis, forecasting and decision-making. Grey entropy theory is used to

6 determine the correlation degree among different factors through calculating the grey

7 correlation degree and the grey entropy correlation degree. The main factors and the

8 secondary factors are divided by analyzing the relationship between the various

9 factors and the system development situation, which play a positive role in guiding

10 the system development [25].

11 Therefore, the grey correlation entropy analysis method was introduced in this

12 paper. In order to find out the main factors influencing the activation energy, the

13 correlation extent between activation energy and asphalt microscopic components,

14 asphalt molecular structure was analyzed based on grey entropy theory analysis

15 method. And analyzing the main factors affecting activation energy of asphalt can not

16 only help us understand the aging mechanism of asphalt from micro level, but also

17 provide theoretical and technical supports for recycling of SBS modified asphalt 18 pavement.

19 Fig. 1 provides a flow chart of calculation steps about grey entropy theory analysis.

20 The steps of comprehensive evaluation using grey correlation entropy analysis

21 method are as follows [26]:

22 Step 1. Determine the evaluation system and collect evaluation data according to the 
1 evaluation purpose. For example: There are $\mathrm{n}$ factors, each factor has $\mathrm{m}$ levels, and

2 these $\mathrm{n} \times \mathrm{m}$ data can form a matrix, Eq.(11).

$$
\left(X_{1}^{\prime}, X_{2}^{\prime} \cdots, X_{n}^{\prime}\right)=\left[\begin{array}{cccc}
x_{1}^{\prime}(1) & x_{2}^{\prime}(1) & \cdots & x_{n}^{\prime}(1) \\
x_{1}^{\prime}(2) & x_{2}^{\prime}(2) & \cdots & x_{n}^{\prime}(2) \\
\vdots & \vdots & \vdots & \vdots \\
x_{1}^{\prime}(\mathrm{m}) & x_{2}^{\prime}(m) & \cdots & x_{n}^{\prime}(m)
\end{array}\right]
$$

4 Step 2. Determine the reference data sequence, Let $X_{0}^{\prime}=\left(x_{0}^{\prime}(1), x_{0}^{\prime}(2), \cdots x_{0}^{\prime}(m)\right)^{T}$, and

5 the others are compare sequence. Dimensionless processing of data is written down as

6 follows, Eq. (12): $X_{i}=\left(x_{i}(1), x_{i}(2), \cdots x_{i}(m)\right)^{T}, i=1,2, \cdots, n$.

$$
x_{i}(k)=\frac{x_{i}^{\prime}(k)}{x_{i}^{\prime}(1)} \quad \mathrm{i}=0,1, \ldots \ldots, \mathrm{n} ; \mathrm{k}=1,2, \ldots \ldots, \mathrm{m}
$$

Step3. Determine $\quad \min _{i} \min _{k}\left|x_{0}(k)-x_{i}(k)\right| \quad$ and $\quad \max _{i} \max _{k}\left|x_{0}(k)-x_{i}(k)\right|$.

$9 \min _{i} \min _{k}\left|x_{0}(k)-x_{i}(k)\right|$ is the minimum difference and $\max _{i} \max _{k}\left|x_{0}(k)-x_{i}(k)\right|$ is the maximum difference. Then calculate the correlation coefficient $\xi_{i}(k)$ and grey

11 correlation degree $r_{0 i}$ according to Eqs. (13) and (14).

$$
\begin{gathered}
\xi_{i}(k)=\frac{\min _{i} \min _{k}\left|x_{0}(k)-x_{i}(k)\right|+\rho \max _{i} \max _{k}\left|x_{0}(k)-x_{i}(k)\right|}{\left|x_{0}(k)-x_{i}(k)\right|+\rho \cdot \max _{i} \max _{k}\left|x_{0}(k)-x_{i}(k)\right|} \\
r_{0 i}=\frac{1}{m} \sum_{k=1}^{m} \xi_{i}(k)
\end{gathered}
$$

Where $\rho$ is resolution coefficient. The smaller the value of $\rho$ is, the greater difference of correlation coefficient has, the stronger ability of distinguish becomes. And the 16 value of $\rho$ usually assumes as 0.5 .

Step 4. Construct grey correlation degree sequence $R_{i}=\left\{\xi_{i}(k) \mid k=1,2, \cdots, m\right\}$. Mapping processing is made for $\mathrm{R}_{\mathrm{i}}$ and the mapping $\mathrm{T}: R_{i} \rightarrow P_{i}$. T is called as distribution mapping of the correlation coefficient $\xi_{i}(k)$, which meets Eq. (15), and $\mathrm{p}_{\mathrm{h}}$ 
1 is called as the density value of distribution.

2

$$
p_{h} \underline{\underline{\Delta}} \frac{\xi_{i}(h)}{\sum_{k=1}^{m} \xi_{i}(k)} \quad p_{h} \in P_{i}, h=1,2, \cdots, m
$$

3 Step 5. Calculate grey correlation entropy and grey entropy correlation degree 4 according to Eq. (16) and Eq. (17).

5

6

7 Where $H\left(R_{i}\right)$ is the grey correlation entropy of $X_{i} ; E_{r}\left(X_{i}\right)$ is the grey entropy 8

$$
\begin{aligned}
H\left(R_{i}\right) & =-\sum_{h=1}^{m} p_{h} \ln p_{h} \\
E_{r}\left(X_{i}\right) & =\frac{H\left(R_{i}\right)}{H_{m}}
\end{aligned}
$$

correlation degree; $H_{m}$ is the maximum of grey correlation entropy and $H_{m}=\operatorname{lnm}$. The correlation between reference data sequence and compare data sequence becomes stronger when grey entropy correlation degree $\mathrm{E}_{\mathrm{r}}\left(\mathrm{X}_{\mathrm{i}}\right)$ increases.

Step 6. Grey correlation degree is a measure of sequence proximity, and grey entropy correlation degree is a measure of sequential equilibrium degree, so equilibrium proximity of sequence is established by the product of grey correlation degree and grey entropy correlation degree, Eq. (18).

$$
w_{i}=E_{r}\left(X_{i}\right) \times r_{0 i}
$$

Where $\mathrm{w}_{\mathrm{i}}$ is the equilibrium proximity between reference data sequence and the $i^{\text {th }}$ compare data sequence. The greater the value of $\mathrm{w}$ is, the more balanced relationship of evaluation object has and the better correlation the reference object has. 


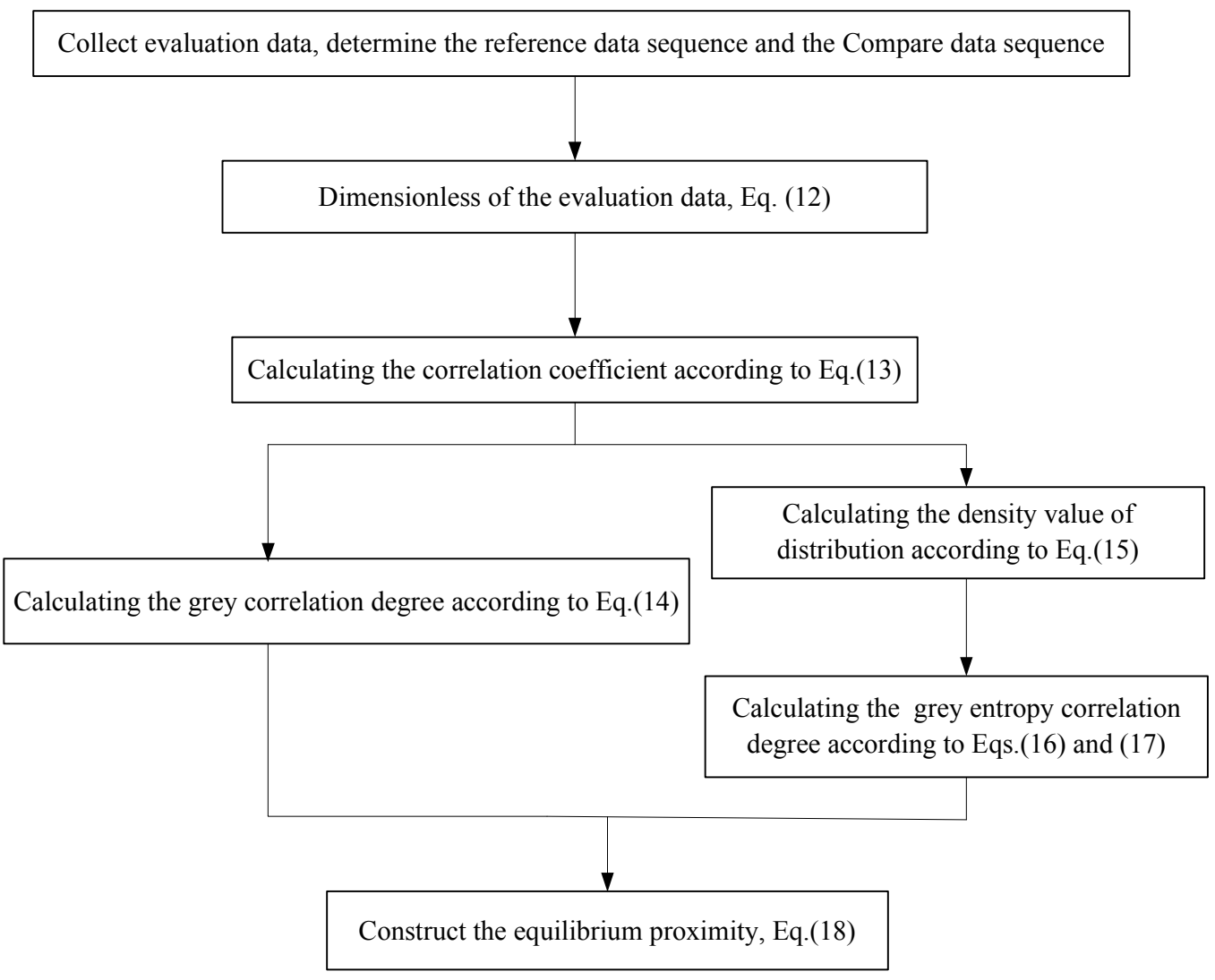

Fig.1 The procedure of calculation steps about grey entropy theory analysis

\section{Test results and discussion}

5.1 Aging reaction kinetics analysis based on complex modulus

6 The complex modulus of asphalt under different aging conditions was tested by 7 dynamic rheological shear apparatus. The complex modulus of asphalt with different 8 aging states showed a similar change rule. The complex modulus increased with the

9 increase of frequency, but decreased with the increase of temperature. Based on time temperature equivalence principle, asphalt complex modulus principal curve was 11 obtained by moving the complex modulus curves of asphalt under different

12 temperature and frequencies to the reference temperature at $64^{\circ} \mathrm{C}$. The complex modulus principal curves of original AH90 and original SBS modified asphalt were 
1 shown in Fig.2 and Fig.3, respectively. The shift factors $\alpha(\mathrm{T})$ of asphalt under

2 different temperature can be calculated according to Eq.(7). And results were shown

3 in Table 2.

4

5

6

7 8

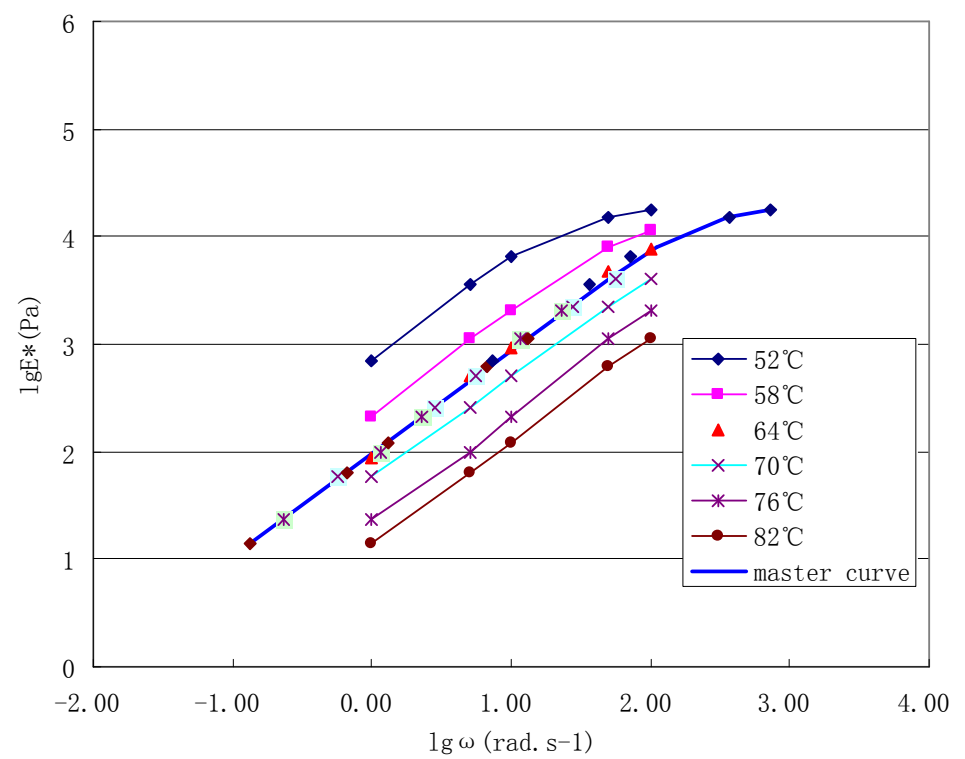

Fig. 2 The master curve of complex modulus of base asphalt

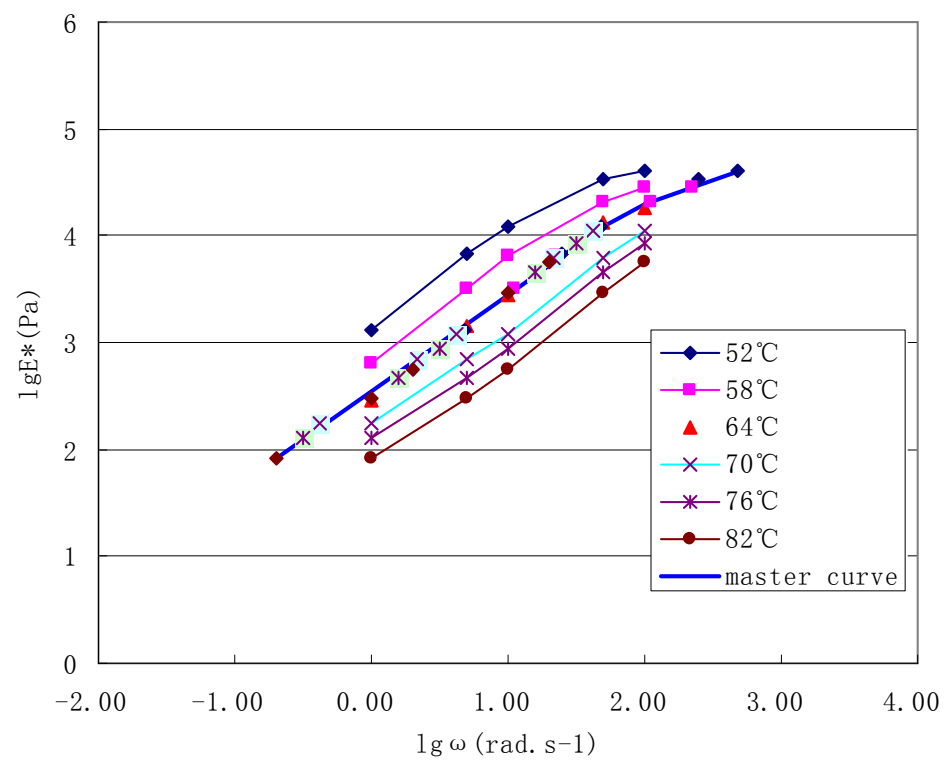

Fig.3 The master curve of complex modulus of SBS modified asphalt 
Table2. The calculation results of shift factors a $(\mathrm{T})$

\begin{tabular}{|l|l|l|l|l|l|l|}
\hline \multirow{2}{*}{ Asphalt types } & \multicolumn{7}{|c|}{ Temperature $\left({ }^{\circ} \mathrm{C}\right)$} \\
\cline { 2 - 8 } & 52 & 58 & 64 & 70 & 76 & 82 \\
\hline AH90-Ori & 7.296703 & 2.263736 & 1 & 0.56044 & 0.230769 & 0.131868 \\
\hline AH90-RTFOT & 6.311321 & 2.490566 & 1 & 0.429245 & 0.198113 & 0.099057 \\
\hline AH90-PAV & 6.397059 & 2.500000 & 1 & 0.431373 & 0.200980 & 0.102941 \\
\hline SBS modified asphalt-Ori & 4.961268 & 2.228873 & 1 & 0.464789 & 0.232394 & 0.130282 \\
\hline SBS modified asphalt- RTFOT & 4.875728 & 2.23301 & 1 & 0.423301 & 0.312621 & 0.200000 \\
\hline SBS modified asphalt- PAV & 4.804074 & 1.787745 & 1 & 0.433953 & 0.142932 & 0.071307 \\
\hline
\end{tabular}

2

3 According to Arrhenius equation Eq.(6), draw a line $\ln \alpha(\mathrm{T})-1 / \mathrm{T}$, whose slope is

4 equal to $\mathrm{Ea} / \mathrm{R}$ and intercept is equal to $-\ln \mathrm{A}$. The regression relation between $\ln \alpha(\mathrm{T})$

5 and 1/T of original base asphalt and original SBS modified asphalt are shown in Fig.4

6 and Fig.5, respectively. Then the activation energy Ea and pre-exponential factor A of

7 asphalt under different aging states were obtained as shown in Table 3.

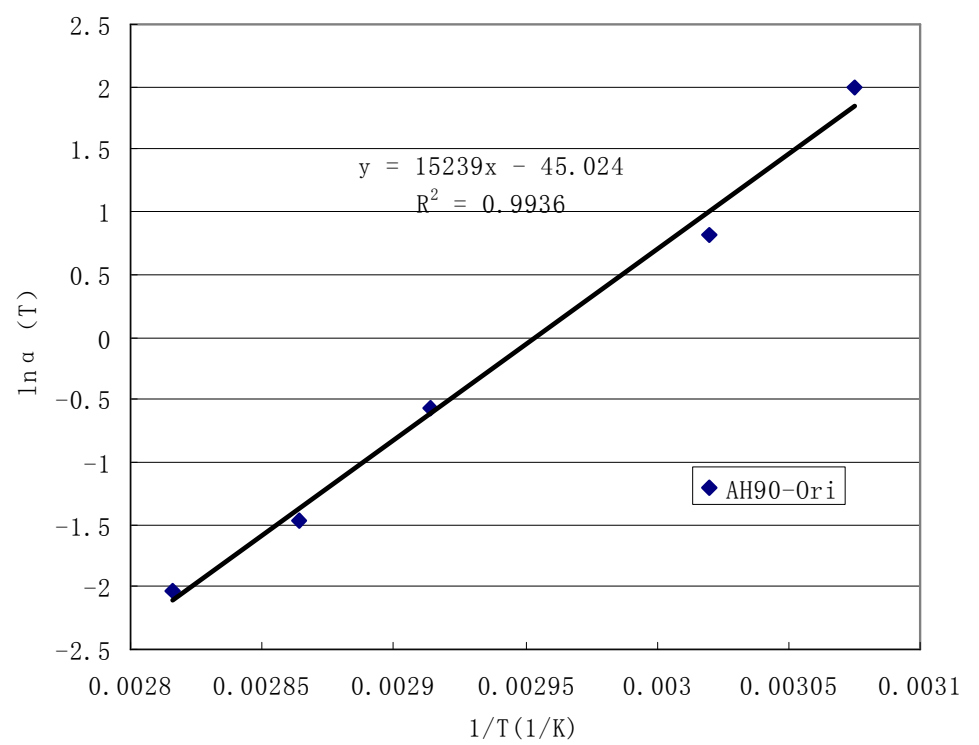

8

$9 \quad$ Fig.4 The relationship between $\ln \alpha(\mathrm{T})$ and 1/T of base asphalt 


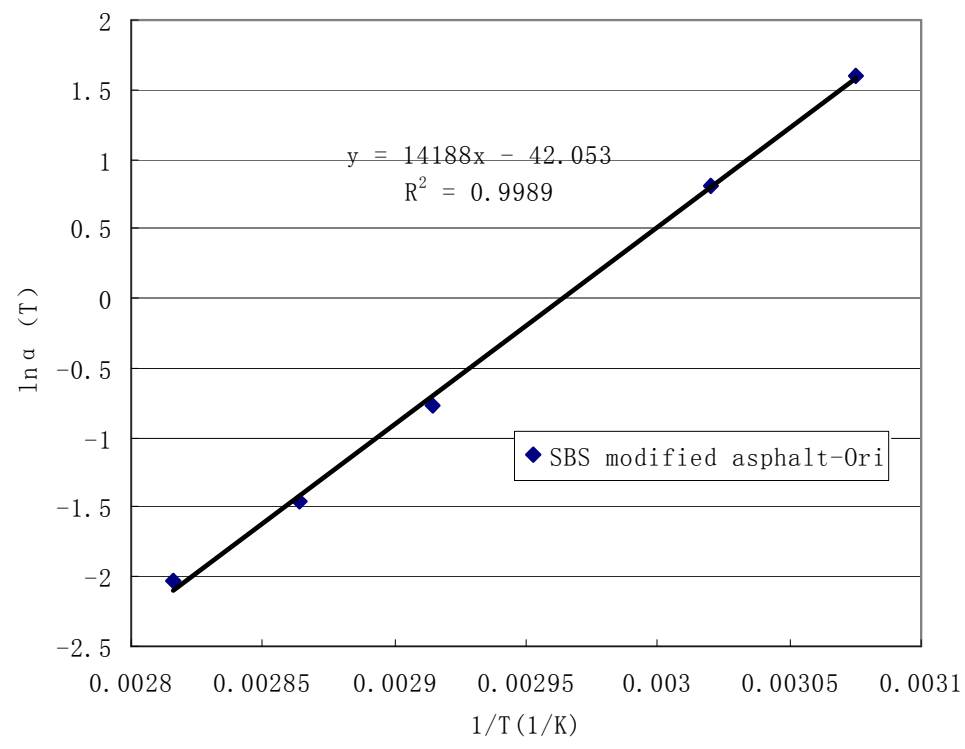

1

Fig.5 The relationship between $\ln \alpha(\mathrm{T})$ and 1/T of SBS modified asphalt

3

4

Table3. The chemical reaction kinetic parameters of asphalt

\begin{tabular}{|l|l|l|l|}
\hline Asphalt types & $\begin{array}{c}\text { Activation energy } \\
\mathrm{E}_{\mathrm{a}}(\mathrm{kJ} / \mathrm{mol})\end{array}$ & $\begin{array}{l}\text { pre-exponential factor } \\
\ln \mathrm{A}\end{array}$ & $\begin{array}{l}\text { correlation coefficient } \\
\mathrm{R}^{2}\end{array}$ \\
\hline AH90-Ori & 126.697 & 45.024 & 0.9936 \\
\hline AH90-RTFOT & 133.905 & 47.721 & 0.9993 \\
\hline AH90-PAV & 133.240 & 47.472 & 0.9989 \\
\hline SBS modified asphalt-Ori & 117.959 & 42.053 & 0.9989 \\
\hline SBS modified asphalt- RTFOT & 104.698 & 37.247 & 0.9814 \\
\hline SBS modified asphalt- PAV & 133.772 & 47.923 & 0.9952 \\
\hline
\end{tabular}

5

6 In the research of aging process of asphalt, activation energy is always regarded as

7 a constant. However, it has been found that activation energy of SBS modified asphalt

8 is various under different aging stages from the calculation in Table 3. Activation

9 energy of SBS modified asphalt after long-term aging increased by $27.8 \%$ compared 
1 with the asphalt after short-term aging.

2 From Eq. (5), it is not difficult to find that the greater the activation energy of

3 asphalt is, the smaller the chemical reaction rate shows and the worse the temperature

4 sensitivity displays. The results of Table 3 show that the aging rate of asphalt under

5 PAV aging condition becomes smaller and the temperature sensitivity becomes worse

6 due to greater activation energy. This is mainly because SBS modified asphalt

7 becomes stiffer and gradually loses activated ingredients after long-term aging. Then

8 the aging rate is restricted to a given temperature. In addition, greater activation

9 energy means a greater chemical reaction rate gradient when the test temperature

10 increases or reduces to a certain degree. Then the features of stiffer will limit the

11 adaptability of asphalt in a certain temperature interval. Therefore, the temperature

12 sensitivity becomes worse, though the aging rate of SBS modified asphalt becomes

13 smaller after long-term aging.

14 Table 3 illustrates that activation energy of AH90 bitumen increases with deepening

15 of aging. However, activation energy of original asphalt decreases and temperature

16 sensitivity increases with the addition of SBS modifier. This is mainly because SBS

17 modifier will disperse into micron grade granules under the high speed shearing

18 action, which increases the specific surface area of SBS modifier. Swelling and

19 plasticizing effects of SBS modifier change the colloidal structure after absorbing the

20 small molecules in asphalt, and three-dimensional network of SBS limits the

21 movement of asphalt colloid particle. As a result, the SBS needs to absorb more

22 energy when the SBS itself deforms or moves [27]. But in the aging process, 
1 activation energy of SBS modified asphalt decreases firstly, then increases, and finally

2 closes to AH90 matrix asphalt. It may be the consequence of synthetic action between

3 medium-sized molecules produced in the aging process and three-dimension network

4 structure under the action of RTFOT, which contribute to a better colloidal stability.

5 However, with further aging of SBS modified asphalt, SBS continued disintegrating

6 and producing much small molecule structures, leading to the temperature sensitivity

7 of SBS modified asphalt closing to the base asphalt after long-term aging.

8 In order to further reveal the nature of activation energy change during asphalt

9 aging, two Microscopic analysis methods, component analysis and GPC analysis,

10 were used to study the change of asphalt composition and molecular structure during

11 the process of aging.

12

\subsection{Component analysis results}

14 Fig.6 represents four component analysis results of AH90 and SBS modified asphalt on different aging stages. Compared with AH90 asphalt, the saturate content significantly decreased along with the addition of SBS modifier into original asphalt.

17 Additionally, the change of aromatics content was not obvious, the resins and asphaltene content increased and the colloidal unstable index decreased obviously for

19 SBS modified asphalt binder. It is the changes in microscopic components that lead to

20 the significant differences of rheological properties and anti-aging performance for

21 SBS modified asphalt compared with AH90 asphalt. 


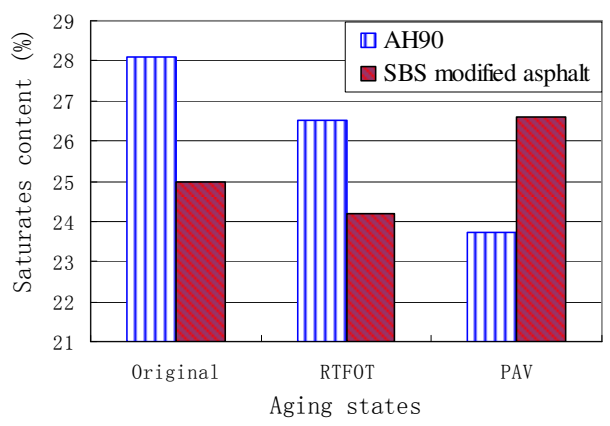

(a) Saturates content

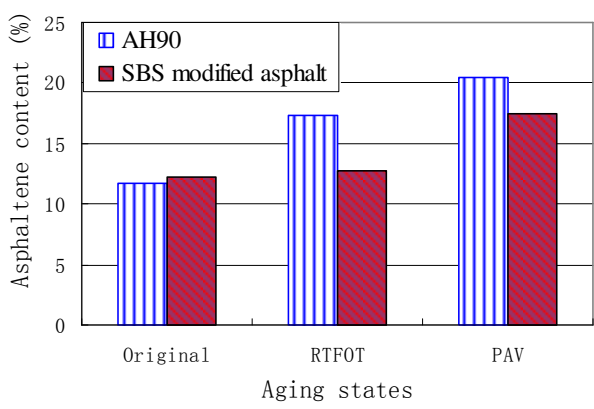

(b) Asphaltene content

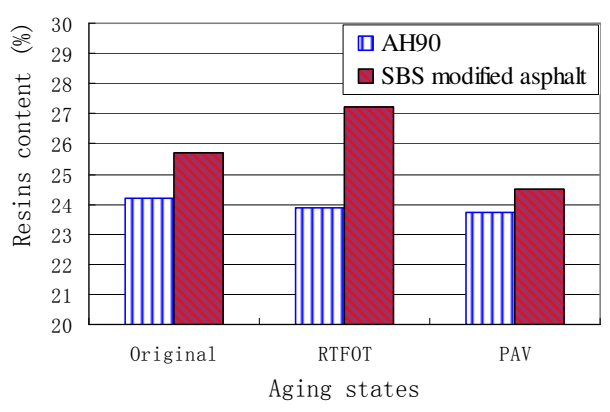

(c) Resins content

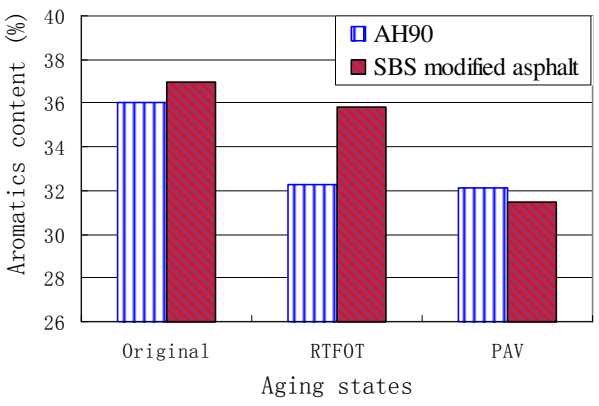

(d) Aromatics content

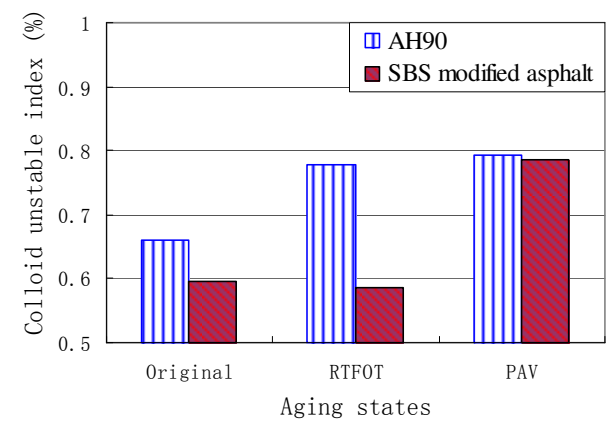

(e) Colloid unstable index

Fig.6 indicates that saturate content and aromatics content of base asphalt will significantly decline and asphaltene content will substantially improve during the aging process. Both the two phenomena will lead to the increase of the colloidal unstable index and the activation energy. When SBS modified asphalt experiences 
1 short-term aging, the saturates content will slightly decrease and the resins content

2 will substantially increase because of further fusion reaction between asphalt and

3 modifier. Therefore, the colloidal unstable index of SBS modified asphalt under

$4 \quad$ RTFOT aging condition will substantially decrease, which will lead to a reduction of

5 activation energy and an improvement of temperature sensitivity. However, after

6 long-term aging, the saturates content of SBS modified asphalt will increase

7 enormously resulting from the degration of SBS modifiers. Furthermore, the vast

8 saturate content and aromatics content will be transformed into asphaltene content,

9 resulting in a substantial increase caused by the unstable colloidal index of SBS

10 modified asphalt. All these varying patterns of components analysis are consistent

11 with the conclusion of the literature [28]. These also explain why the activation

12 energy of SBS modified asphalt after long-term aging basically approach to base

13 asphalt and even more lose the modification effects.

\subsection{GPC analysis results}

16 GPC is a kind of technology used to measure relative molecular mass. Fig.7 and

17 Fig.8 respectively shows the GPC chromatograms of base asphalt AH90 and SBS modified asphalt under different aging stages. The area under the curve represents

$19100 \%$ of the binder's molecules injected into the GPC system. Researchers have 20 classified binder constituents into several groups by molecular size [29-30]. In this 21 study, a chromatographic profile was partitioned into 9 slices, which were further 22 divided into three parts, namely large molecular size (LMS), medium molecular size 
1 (MMS) and small molecular size (SMS), corresponding to slices with the sizes of 1-4,

2 5-7, and 8-9, respectively. Defining LMS part as front 4 slices is based on the

3 previous study, in which LMS was defined as the area from elution start to the elution

4 time slightly over $1 / 3$ of total elution time [31]. The elution of sample is detected in

5 milli-volt $(\mathrm{mV})$ as shown in Fig. 7and Fig. 8. Relative quantity (the area under the

6 curve) by percentage of each part to for asphalt binder under different aging states

7 was respectively calculated, as shown in Table 4.

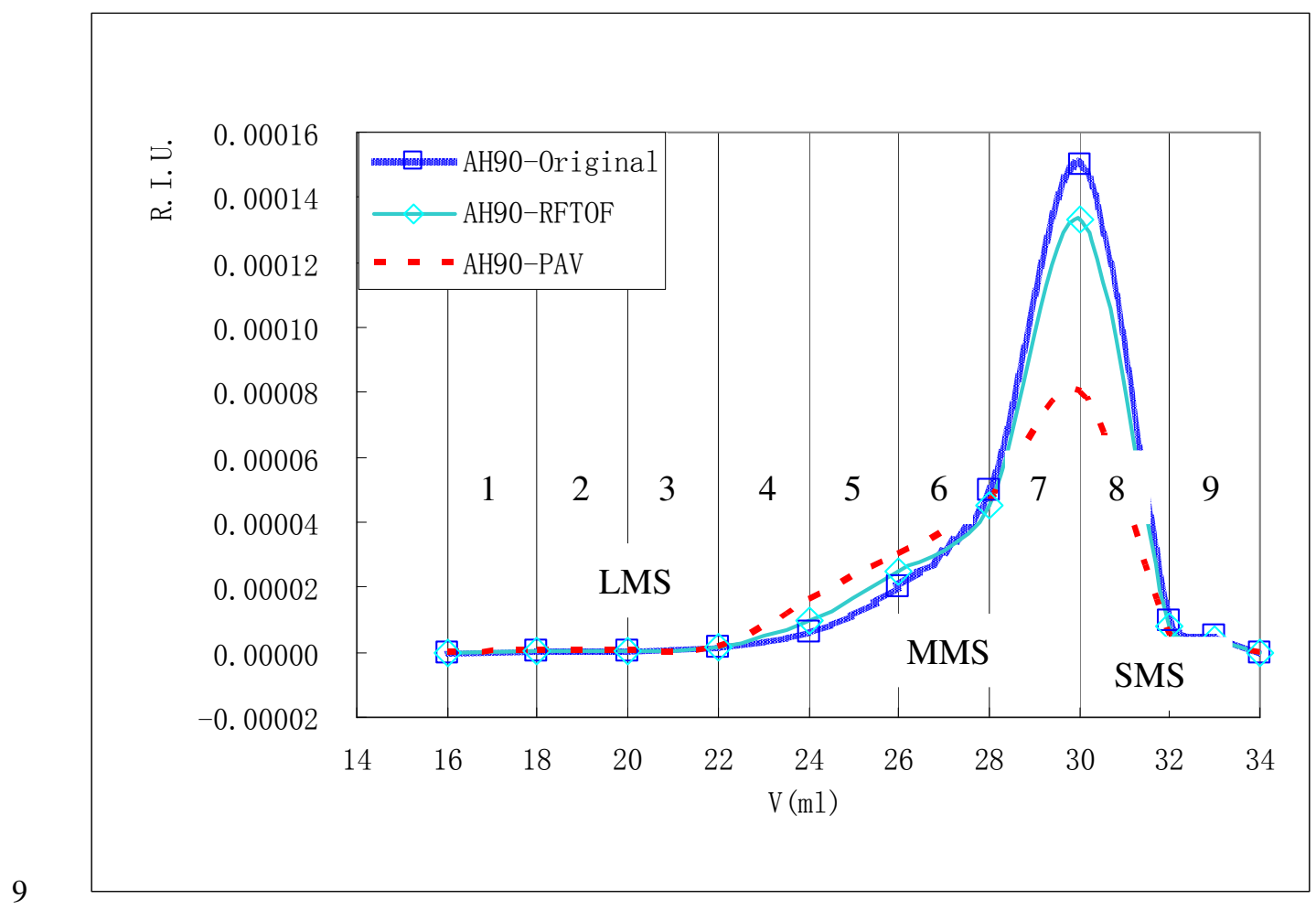




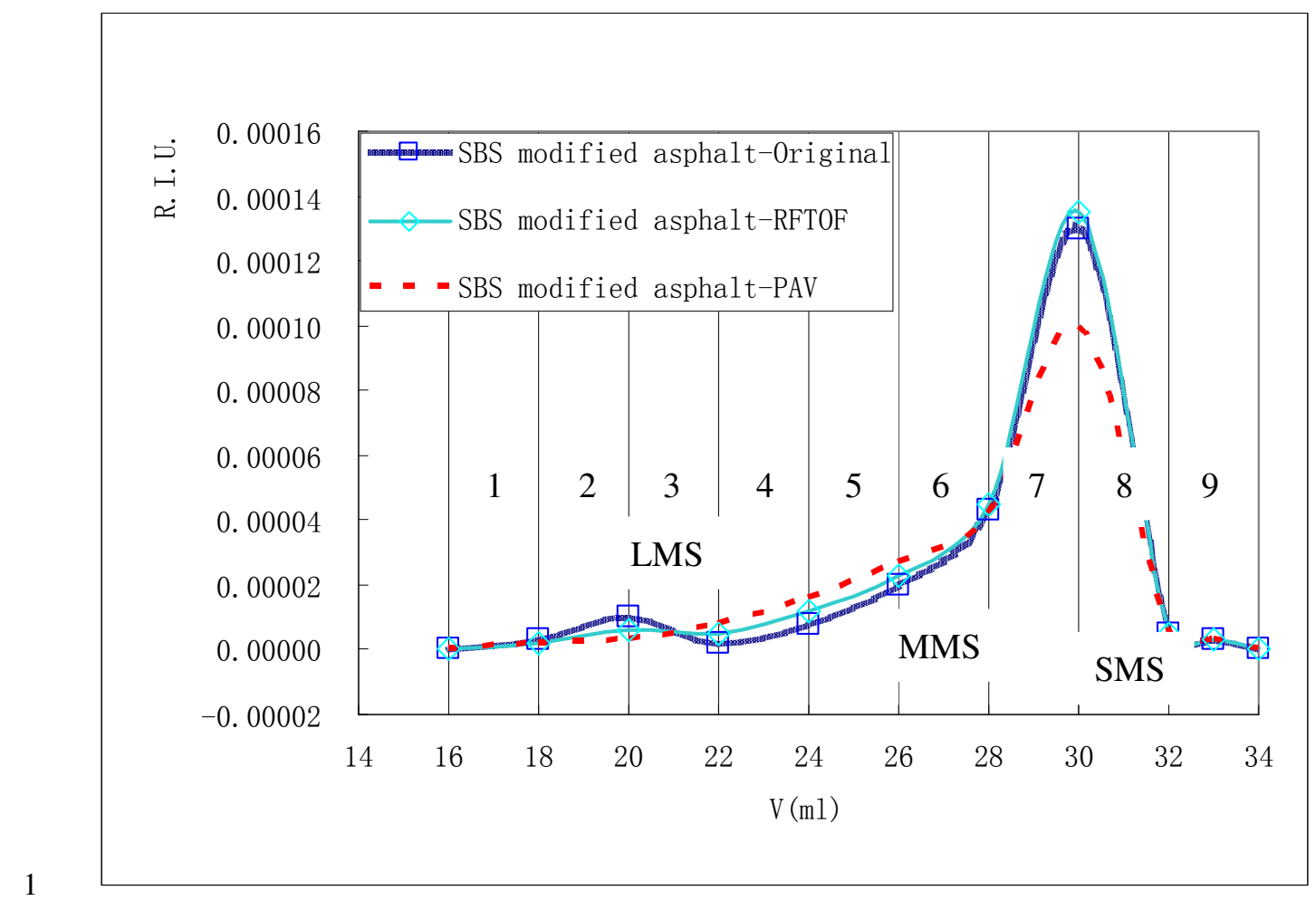

Fig. 8 GPC chromatogram of SBS modified asphalt binder

Table 4. The results of GPC analysis

\begin{tabular}{|l|l|l|l|l|l|l|}
\hline Asphalt types & LMS(\%) & MMS(\%) & SMS(\%) & Mn & Mw & d \\
\hline AH90-Ori & 6.55 & 56.72 & 36.73 & 666.1 & 3855 & 5.787 \\
\hline AH90-RTFOT & 10.00 & 56.27 & 33.73 & 735.7 & 4510 & 6.130 \\
\hline AH90-PAV & 16.82 & 57.32 & 25.86 & 768.7 & 4872 & 6.338 \\
\hline SBS modified asphalt-Ori & 8.55 & 59.82 & 31.63 & 835.2 & 14794 & 17.713 \\
\hline SBS modified asphalt- RTFOT & 8.60 & 60.57 & 30.83 & 949.7 & 12314 & 12.996 \\
\hline SBS modified asphalt- PAV & 9.53 & 61.87 & 28.60 & 988.5 & 11137 & 11.267 \\
\hline
\end{tabular}

5 Note : $\mathrm{Mn}$-number-average molecular weight; $\mathrm{Mw}$-weight-average molecular

6 weight; d-dispersion.

7

8 The results of Fig.7 show that the small molecules of base asphalt gradually transform 
1 into large molecules during the aging process. And in Fig.8, distinct double peak

2 phenomenon can be found in the GPC chromatogram of original SBS modified

3 asphalt. This double peak are also detected by the references [23,24]. According to the

4 principal of GPC, the first elution content (the first peak of Fig.7 16ml-22ml) is SBS

5 modifier which has bigger molecular weight, and the second elution content (the

6 second peak of Fig.8) is the small molecular substances in asphalt. SBS modifier will

7 degrade into small molecules with the deepening of aging, which will lead to an

8 increase of molecular composition with the leaching volume at $22 \mathrm{ml}-24 \mathrm{ml}$. And in

9 order to understand the molecular weight changes of SBS modifier, the big molecular

10 substances (elution content $16 \mathrm{ml}-22 \mathrm{ml}$ ) were separated and calculated, as shown in

$11 \quad$ Table 5.

Table 5. Molecular weight calculation results of SBS modifier

\begin{tabular}{|l|l|l|l|}
\hline Asphalt types & Mn & Mw & d \\
\hline SBS modified asphalt-Ori & 159321 & 186328 & 1.169513 \\
\hline SBS modified asphalt-RTFOT & 118938 & 153470 & 1.290336 \\
\hline SBS modified asphalt-PAV & 69153 & 110082 & 1.591862 \\
\hline
\end{tabular}

14 Generally the number-average molecular weight reflects the change trend of medium

15 or small molecules in asphalt, while the weight-average molecular weight reflects the

16 content of macromolecular in asphalt. The results of Table 4 show that the

17 number-average molecular weight of SBS modified asphalt increases, but the

18 weight-average molecular weight constantly decreases with the deepening of aging,

19 which mainly results from the splitting of SBS modifiers. The results of Table 5 
1 illustrate that the decreasing trend of molecular weight of SBS modifier is obvious in

2 the process of aging. The weight-average molecular weight of SBS modifier

3 respectively decreases by $18 \%$ and $41 \%$ after short-term aging and long-term aging.

4 The number-average molecular weight of SBS modifier after long-term aging is close

5 to half of its original value, which means SBS modifier has suffered serious

6 degradation and it seriously affects the SBS modified asphalt properties. All these

7 phenomena are consistent with the conclusion of the literature [20]. However,

8 compared with the number-average molecular weight, the change of weight-average

9 molecular weight of base asphalt is more sensitive. The asphalt aging reaction mainly

10 includes dissociation and condensation. The condensation will generate more and

11 more macromolecular structure, which lead to greater weight-average molecular

12 weight of base asphalt, while the dissociation will produce more and more small

13 molecules with low boiling point. However, the condensation plays a leading part in

14 the aging process of base asphalt and only small amounts of dissociation will occur,

15 which finally leads to increase the dispersion of base asphalt after aging.

\subsection{Grey entropy theory analysis results}

According to the grey correlation entropy analysis method, the activation energies $\left(\mathrm{X}_{0}\right)$ is used as reference sequence in this paper. Saturates content $\left(\mathrm{X}_{1}\right)$, asphaltenes

20 content $\left(\mathrm{X}_{2}\right)$, resins content $\left(\mathrm{X}_{3}\right)$, aromatics content $\left(\mathrm{X}_{4}\right)$, colloidal unstable index $\left(\mathrm{X}_{5}\right)$,

21 large molecular size structure content $\left(\mathrm{X}_{6}\right)$, medium molecular size structure 22 content $\left(\mathrm{X}_{7}\right)$ and small molecular size structure content $\left(\mathrm{X}_{8}\right)$ served as compare 
1 sequence. Then the influence of micro components and molecular structures on

2 activation energy was analyzed as the six steps in section 4.4. The results of grey

3 correlation entropy analysis are shown in Table 6.

Table 6. The results of grey correlation entropy analysis

\begin{tabular}{|l|l|l|l|}
\hline Compare sequence & Grey correlation degree & Entropy correlation & Equilibrium \\
\hline Saturates content $(\mathrm{X} 1)$ & 0.90409 & $\mathrm{E}_{\mathrm{r}}\left(\mathrm{X}_{\mathrm{i}}\right)$ & ${\text { proximity extent } \mathrm{w}_{\mathrm{i}}}$ \\
\hline Asphaltenes content (X2) & 0.73575 & 0.99827 & 0.90253 \\
\hline Resins content $(\mathrm{X} 3)$ & 0.88837 & 0.98718 & 0.72632 \\
\hline Aromatics content $(\mathrm{X} 4)$ & 0.86366 & 0.99687 & 0.88560 \\
\hline Colloidal unstable index $(\mathrm{X} 5)$ & 0.90752 & 0.99844 & 0.86231 \\
\hline LMS structure content $(\mathrm{X} 6)$ & 0.64740 & 0.99881 & 0.90645 \\
\hline MMS structure content $(\mathrm{X} 7)$ & 0.90733 & 0.97453 & 0.63091 \\
\hline SMS structure content (X8) & 0.86036 & 0.99784 & 0.90537 \\
\hline
\end{tabular}

6 Through comparing the equilibrium proximity $\mathrm{w}_{\mathrm{i}}$ according to Table 6 , it can be

7 shown that $\mathrm{X} 5>\mathrm{X} 7>\mathrm{X} 1>\mathrm{X} 3>\mathrm{X} 4>\mathrm{X} 8>\mathrm{X} 2>\mathrm{X} 6$. The results indicate that the factors

8 have closely correlation with activation energy include colloidal unstable index,

9 medium molecular size structure content and saturates content. It is conductive to

10 reduce activation energy and improve asphalt temperature sensitivity with the

11 decrease of colloidal unstable index and saturates content, the increase of medium

12 molecular size structure content. The addition of SBS modifier not only reduces the

13 saturates content but also improves the content of MMS structure, which decreases the

14 activation energy of SBS modified asphalt and enhances the stability of the asphalt. 
1 But activation energy of SBS modified asphalt has changed under aging process. The

2 variation of saturates content of SBS modified asphalt during short-term aging is

3 reduced. In addition, the formation of MMS structure due to the degradation of SBS

4 modifiers decreases the activation energy of SBS asphalt after short-term aging and

5 improves the temperature sensitivity. However, after long-term aging, the saturates

6 content of SBS modified asphalt increases significantly and the resins content

7 decreases obviously. As a consequence, the activation energy of SBS modified asphalt

8 increases, the temperature sensitivity of SBS modified asphalt tend to be closer to

9 base asphalt. Therefore, the modification effect of SBS modified asphalt is not evident

10 after long-term aging.

11

12

\section{Conclusions}

This paper analyzed the activation energy of asphalt under different aging conditions based on the principle of chemical reaction kinetics. Then component analysis and GPC were respectively used to study the change of asphalt composition and molecular structure during aging process. In addition, the correlation between activation energy and asphalt microscopic components, asphalt molecular structure was also analyzed based on grey entropy theory analysis method. The following conclusions can be drawn.

(1) The calculation results of activation energy illustrate that the activation energy of asphalt is not as generally believed as a constant, but it changes along with aging. The activation energy of base asphalt will increase during aging process. However, for SBS modified asphalt, the activation energy will decrease firstly and then increase. 
1 Therefore, the activation energy can be used to evaluate the aging degree of SBS

2 modified asphalt as one evaluation index.

3 (2) The aging of SBS modified asphalt is formed by both aging of base asphalt and

4 SBS modifier. In the process of aging, SBS modifiers are gradually degraded into

5 small molecules and SBS modified network structures gradually lose effectiveness.

6 SBS modified asphalt under different aging degree shows different activation energy

7 value because of the differences of composition and molecular structure. SBS

8 modified asphalt after short-term aging will degrade to more medium molecular size

9 structures, which make the activation energy become lower. But the activation energy

10 of SBS modified asphalt after long-term aging increases and temperature sensitivity

11 approximate to base asphalt due to the significant increase of saturates content and

12 decrease of resins content. The SBS modifiers after long-term aging have almost

13 degraded completely and lost modification effect.

14 (3) The factors that have closely correlation with activation energy contain colloidal

15 unstable index, medium molecular size structure content, saturates content and resins

16 content. The activation energy is reduced and asphalt temperature sensitivity is

17 improved with the increase of medium molecular size structure content, the decrease

18 of saturates content and the increase of resins content. This also explains why

19 activation energies are different under different aging states.

20 (4) SBS modified asphalt under serious aging condition will lose modification effect,

21 which will make the activation energy of SBS modified asphalt approximate to

22 ordinary unmodified asphalt. Therefore, SBS modified asphalt under serious aging 
1 condition is unsuitable for directly modified recycling without the second

2 modification. However, SBS modified asphalt under serious aging condition can be

3 applied to recycled asphalt mixture as aged ordinary unmodified asphalt. It is a good

4 recommendation that the aging degree of SBS modified asphalt should be evaluated

5 before recycling SBS modified asphalt mixture.

6 (5) However, results and analyses provided in this paper are only based on one base

7 asphalt binder and one SBS modified binder. A further study on more kinds of asphalt

$8 \quad$ will be the focus of our next work.

9

21 Transportation Institute.

\section{Acknowledgement} 51250110075 and U1134206).

\section{References}

This research is sponsored by the National Science Foundation (CMMI-0408390, CMMI-0644552), the American Chemical Society Petroleum Research Foundation (PRF-44468-G9), and National Natural Science Foundation of China (51050110143,

[1] Mastrofini D, Scarsella M. The application of rheology to the evaluation of bitumen ageing. Fuel 2000;79:1005 - 1015.

[2] Woo, W. J., Ofori-Abebresse, E., Chowdhury, A., Hilbrich, J., Kraus, Z., Martin, A. E., and Glover, C. J. (2007). Polymer modified asphalt durability in pavements. Publication FHWA/TX-07/0-4688-1, FHWA/TX-07. College Station, TX: Texas

[3] Xue Luo, Ph.D., Rong Luo, Ph.D., P.E., M.ASCE, Robert L. Lytton, Ph.D., P.E., 
1 F.ASCE. Energy-Based Mechanistic Approach to Characterize Crack Growth of

2 Asphalt Mixtures. J. Mater. Civ. Eng. 2013;25:1198-1208.

3 [4] Lubinda F. Walubita, Geoffrey S. Simate, Edward Ofori-Abebresse, Amy Epps

4 Martin,Robert L. Lytton, Luis E. Sanabria. Mathematical formulation of HMA crack

5 initiation and crack propagation models based on continuum fracture-mechanics and

$6 \quad$ work-potential theory. International Journal of Fatigue 2012; 40: 112 - 119.

7 [5] Buttlar, W. G., Bozkurt, D., Al-Khateeb, G.G., and Waldhoff, A.S., Understanding 8 Asphalt Mastic Behavior Through Micromechanics. Transportation Research Record $9 \quad 1999 ; 1681: 157-169$.

10 [6] James W. Levis , Morton A. Barlaz,Akhtar Tayebali. Quantifying the greenhouse 11 gas emission reductions associated with recycling hot mix asphalt. Road Materials 12 and Pavement Design 2011;12(1):57-77.

13 [7] Gordon D.Airey. Rheological properties of styrene butadiene styrene polymer 14 modified road bitumens. Fuel, 2003;82:1709-1719.

15 [8] Pang Ling, Zhao Zhijun, Ma Lixin, Zhu Guojun. Aging evaluations of AH- 90 16 and PG70-28 polymer modified asphalt. JOURNAL OF WUHAN UNIVERSITY OF TECHNOLOGY 2007;29(9):1-4.(in Chinese)

[9] Mohammd Nahid Siddiqui, Mohammad Farhat Ali,John Shirokoff. Use of X-ray diffraction in assessing the aging pattern of asphalt fractions. Fuel 2002;81:51-58. [10] Vargas, María A., et al. Asphalt/polyethylene blends: Rheological properties, 21 microstructure and viscosity modeling. Construction and Building Materials 2013;45: $22 \quad 243-250$. 
1 [11] Mturi, Georges AJ, Johan O'Connell, and Joseph K. Anochie-Boateng.

2 Limitations of current South African test methods for bituminous binders.

3 Construction and Building Materials 2013;45: 314-323.

4 [12] Lu, Xiaohu, and Ulf Isacsson. Effect of ageing on bitumen chemistry and

5 rheology. Construction and Building Materials 2002;16.1: 15-22.

6 [13] Herrington P, James B, Henning T F. Model for long term bitumen oxidation.

7 ARRB Conference, 26th, 2014, Sydney, New South Wales, Australia. No. 1.1. 2014.

8 [14] Jin, Xin, et al. Fast-rate-constant-rate oxidation kinetics model for asphalt

$9 \quad$ binders. Industrial \& Engineering Chemistry Research 2011;50.23: 13373-13379.

10 [15] Yang Kang, Fei Wang, Zhiming Chen. Reaction of asphalt and maleic anhydride:

11 Kinetics and mechanism. Chemical Engineering Journal 2010;164: 230 - 237.

12 [16] R. Han, X. Jin and C. J. Glover. Oxygen Diffusivity in Asphalts and Mastics.

13 Petroleum Science and Technology 2013; 31:1563-1573.

14 [17] Fabrikant I I, Hotop H. On the validity of the Arrhenius equation for electron

15 attachment rate coefficients[J]. The Journal of Chemical Physics, 2008, 128(12).

16 [18] Ruan Y, Davison RR, Glover CJ. The effect of long-term oxidation on the 17 rheological properties of polymer modified asphalts. Fuel 2003;82:1763 - 73.

18 [19] Maninder Singh, Praveen Kumar, Mannan Ram Maurya. Effect of aggregate

19 types on the performance of neat and EVA-modified asphalt mixtures. International

20 Journal of Pavement Engineering 2014;15(2):163-173.

21 [20] Ma, Tao. Research on hot in-place recycling technology of SMA pavement.

22 Doctoral dissertation. Southeast University,2009. 
1 [21]D. Q. Sun, L. W. Zhang. A quantitative determination of polymer content in SBS

2 modified asphalt. Part I: State of the Art.Petroleum Science and Technology, $3 \quad 2013 ; 31: 2636-2642$.

4 [22]Ala R. Abbas, Umme Amina Mannan, Samer Dessouky. Effect of recycled 5 asphalt shingles on physical and chemical properties of virgin asphalt binders.

6 Construction and Building Materials 2013;45:162 - 172.

7 [23] Sun L, Wang Y, Zhang Y. Aging mechanism and effective recycling ratio of SBS 8 modified asphalt. Construction and Building Materials 2014; 70: 26-35.

9 [24] Daranga, Codrin. Characterization of aged polymer modified asphalt cements for 10 recycling purposes. Doctoral dissertation. Louisiana State University, 2005.

11 [25] Igor Pantic,1Senka Pantic. Germinal center texture entropy as possible indicator 12 of humoral immune response:immunophysiology viewpoint. Mol Imaging Biol $13 \quad 2012 ; 14: 534-540$.

14 [26] Qiu Heting, Li Xuemei, and Li Xuewei. Combined evaluation of logistics service 15 providers based on correlation[C]// Zhang, Z. 2nd International Conference on 16 Logistics, Informatics and Service Science (LISS). Beijing: Beijing Jiaotong Univ, 17 Sch Econ \& Management ,2013:1077- 1082.

18 [27]Rossi D, Filippi S, Merusi F, et al. Internal structure of bitumen/polymer/wax 19 ternary mixtures for warm mix asphalts. Journal of Applied Polymer Science, 2013, 20 129(6):3341-3354.

21 [28] Ma, Tao, and Hao Yuan. Aging behavior characterization of SBS-modified 22 asphalt for recycling purpose. Bridges 2012;10: 9780784412671-0023. 
1 [29] Kim KW, Burati JL. Use of GPC chromatogram to characterize aged asphalt

2 cement. J Mater Civil Eng ASCE 1993;5(1):41 - 52.

3 [30] Kim KW, Burati JL, Amirkhanian SN. Relation of HP-GPC Profile with

4 Mechanical Properties of AC Mixtures. JMater Civil Eng ASCE 1993;5(4):447 - 59.

5 [31] Kim KW, Burati JL, Park JS. Methodology for defining LMS portion in asphalt 6 chromatogram. J Mater Civil Eng ASCE 1995;7(1):31 - 40.

7 